geführt werden müssen. Die Entwicklung der Zahl der Aerzte in Deutschland seit 1901 ist aus der folgenden Zusammenstellung ersichtlich:

$\begin{array}{cccc} & \text { Zahl der Aerzte } & \begin{array}{c}\text { Zunahme gegen } \\ \text { das Vorjahr }\end{array} & \begin{array}{c}\text { Aerzte auf } \\ 10000 \text { Einw. }\end{array} \\ 1901 & 27978 & -4,92 \\ 1902 & 28860 & 882 & 5,00 \\ 1903 & 29679 & 819 & 5,06 \\ 1904 & 30071 & 392 & 5,06 \\ 1905 & 30655 & 584 & 5,08 \\ 1906 & 30931 & 276 & 5,06 \\ 1907 & 31416 & 485 & 5,07 \\ 1908 & 31640 & 224 & 5,04 \\ 1909 & 31969 & 329 & 5,02 \\ 1910 & 32449 & 480 & 5,03 \\ 1911 & 32835 & 386 & 5,02 \\ 1912 & 33527 & 692 & 5,06 \\ 1913 & 34136 & 609 & 5,11\end{array}$

Unter den 34136 Aerzten sind die Schiffsärzte und die Aerzte in den deutschen Kolonien nicht eingerechnet. Am 1. November 1913 waren von der Hamburg-Amerikalinie 93, vom Norddeutschen Lloyd 75 Schiffsärzte angestellt. In den deutschen Schutzgebieten waren 58 Amtsärzte und mit amtlichen Funktionen betraute Zivilärzte, außerdem 105 Sanitätsoffiziere (42 in Ostafrika, 22 in Südwest, 26 in Kamerun und 15 in Kiautschou).

Der jährliche Abgang an Aerzten durch Tod, durch Verzug ins Ausland etc. beträgt im Durchschnitt etwa 500,1) vom l. November 1912 bis 1. November 1913 verzeichnet der Aerztekalender 444 gestorbene Aerzte; sehr viel größer kann deren Zahl nicht sein, wenn auch angenommen werden muß, daß die Liste nicht ganz vollständig ist. Nimmt man einen jahrlichen Zuwachs von 800000 Einwohnern in Deutschland an (künftig wird er allerdings geringer werden), so erfordern diese etwa 400 Aerzte; beträgt der Zuwachs an Aerzten mehr als 900, so muß die Verhältniszahl der Aerzte rasch zunehmen.

Die Zahl der medizinischen A pprobationen ist im Jahre 1911/12 wieder beträchtlich gestiegen, und zwar um 185, also erheblich mehr als im Vorjahre, in dem die Zunahme 102 betrug. Es erhielten die Approbation:

$\begin{array}{rrrr}1906-1907 & 553 & 1909-1910 & 945 \\ 1907-1908 & 829 & 1910-1911 & 1047 \\ 1908-1909 & 942 & 1911-1912 & 1232\end{array}$

Die Zahl der medizinischen Vorprüfungen nimmt seit 6 Jahren ungemein rasch zu; während diese 1906/07 1038 betrug, ist sie $1907 / 08$ auf $1335,1908 / 09$ auf $1461,1909 / 10$ auf $1713,1910 / 11$ auf 1858 und 1911/12 sogar auf 2005 angestiegen; unter der letzteren Ziffer sind 101 Ausländer. Es ist also im Jahre 1916 ein Zugang von etwa 2000 jungen Aerzten zu erwarten.

Die Zahl der Medizinstudierenden ist immer noch in $\mathrm{Zu}$. nahme begriffen. Sie war

$\begin{array}{ccc} & \text { Sommerhalbjahr } & \text { Winterhalbjahr } \\ 1905 & 6032 & 6080 \\ 1906 & 6570 & 7219 \\ 1907 & 7574 & 7773 \\ 1908 & 8250 & 8879 \\ 1909 & 9239 & 10263 \\ 1910 & 11125 & 11240 \\ 1911 & 11927 & 12446 \\ 1912 & 13380 & 13904 \\ 1913 & 14750 & -\end{array}$

Auch in Oesterreich ist die Zahl der Medizinstudierenden ungemein rasch gestiegen; sie betrug im Wintersemester 1903/04 2509, im Sommersemester 1913 dagegen 6168. Die Steigerung in Deutschland ist so groß, daß die ebenfalls zunehmende Zahl der Reichsausländel an der Tatsache des ungeheuren Anwachsens der Zahl der deutschen Medizinstudierenden nichts ändert. An den deutschen Universitäten waren Reichsausländer zum Studium der Medizin

$\begin{array}{lcc} & \text { Sommerhalbjahr } & \text { Winterhalbjahr } \\ 1909 & 1091 & 1491 \\ 1910 & 1499 & 1774 \\ 1911 & \text { ca. } 1800 & 1979 \\ 1912 & 2060 & 2278 \\ 1913 & 2164 & -\end{array}$

Die meisten Ausländer sind Russen, es waren 1250 im Sommer 1911 und $1634 \mathrm{im}$ Sommer 1913.

Aus der folgenden Tabelle ist die Zahl der Aerzte in den einzelnen Landesteilen im Vergleich mit dem Vorjahre ersichtlich

\begin{tabular}{|c|c|c|c|c|c|}
\hline & \multirow[t]{2}{*}{ 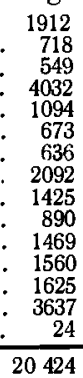 } & \multirow[t]{2}{*}{$\begin{array}{r}1913 \\
734 \\
546 \\
4151 \\
1098 \\
677 \\
646 \\
2094 \\
1425 \\
906 \\
1504 \\
1598 \\
1661 \\
3705 \\
21 \\
20766\end{array}$} & \multirow[t]{2}{*}{ 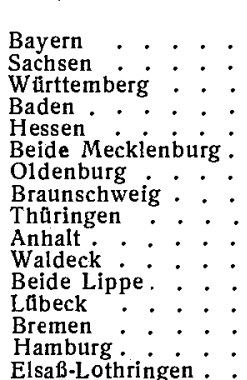 } & \multirow[t]{2}{*}{ 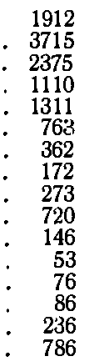 } & \multirow[t]{2}{*}{$\begin{array}{r}1913 \\
3779 \\
2425 \\
1135 \\
1336 \\
766 \\
354 \\
179 \\
284 \\
728 \\
156 \\
53 \\
74 \\
90 \\
255 \\
830 \\
926\end{array}$} \\
\hline Ganz Preuße & & & & & \\
\hline
\end{tabular}

1) Am 1. November 1911 war der Bestand an Aerzten 32835 , das Mittel des Zugangs aus den Approbationen 1910-11 und 1911--12 war 1140 (die Einjührigen Aerzte werden im Reichsmedizinalkalender nicht aufgeführt), der Bestand am 1. November 1912 war 33527 , demnach Abgang 448. Deutschlands um die Mitte dieses Jahres 66835000 , somit kommen auf 10000 Einwohner 5,11 Aerzte. Von der Summe der Aerzte kommen etwa 100 Aerzte in Abzug, die im Sommer in Badeorten, im Winter in anderen Städten die ärztliche Praxis ausüben und daher zweimal auf

\section{Die Aerzte Deutschlands im Jahre 1913.}

Die Zahl der Aerzte in Deutschland beträgt im Jahre 1913 nach dem von Geheimrat Schwalbe herausgegebenen ,Reichsmedizinalkalender für Deutschland auf das Jahr $1914^{\text {" }}$ (Leipzig, G. Thieme, 1914, abgeschlossen am l. November 1913) 34136. Nach dem Statistischen 
Fast überall findet man in Deutschland eine Zunahme der Aerzte, eine Ausnahme machen nur Westpreußen, Provinz Sachsen, Sigmaringen, Mecklenburg, Waldeck, beide Lippe.

Ungemein zahlreich sind die Aerzte in den Großstädten; in der folgenden Zusammenstellung sind zur Berechnung von Verhältnisziffern die ungefähren Einwohnerzahlen der Großstädte am 1. Jnli 1912 und 1913 angegeben.

\begin{tabular}{lcccccc} 
& \multicolumn{2}{c}{ Einwohnerzahlen } & \multicolumn{2}{c}{ Zahl der } & Aerzte & \multicolumn{2}{c}{ auf 10000 Ein- } \\
& \multicolumn{2}{c}{ wohner Aerzte } \\
& 1912 & 1913 & 1912 & 1913 & 1912 & 1913 \\
Großstådte & 14980000 & 15348000 & $14281^{1}{ }^{1}$ & 14772 & 9,5 & 9,6 \\
Uebriges Deutschland & 51323000 & 51487000 & 19246 & 19364 & 3,7 & 3,8
\end{tabular}

Während also die Zahl der Aerzte in den Großstädten um 491 zugenommen hat, ist sie im übrigen Deutschland nur um 118 gewachsen. In Groß-Berlin ist die Zahl der Aerzte jetzt 4151; nimmt man eine jährliche Zunahme der Bevölkerung entsprechend dem Wachstum von 1905 auf 1910 an, so kommen auf 10000 Einwohner 11,09 Aerzte. In den nächstvorhergegangenen Jahren war die Zahl der Aerzte GroßBerlins

$\begin{array}{lcc} & \text { überhaupt } & \text { auf } 10000 \text { Einwohner } \\ 1909 & 3844 & 11,25 \\ 1910 & 3894 & 11,10 \\ 1911 & 3938 & 10,90 \\ 1912 & 4032 & 11,05 \\ 1913 & 4151 & 11,09\end{array}$

In den anderen Großstädten kamen unter Zngrundelegıng der auf die Mitte des Jahres berechneten Bevölkerung Aerzte auf 10000 Einwohner:

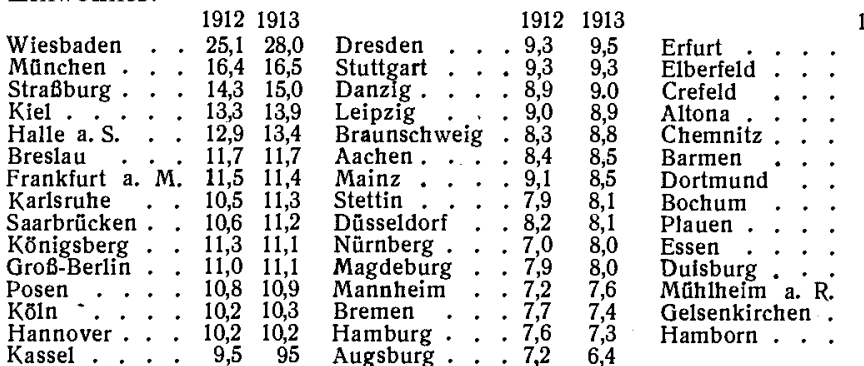

Im allgemeinen zeigen sich in allen Jahren dieselben Verhältnisse; wie sonst fällt die kleine Zahl der Aerzte in den Industriestädten des Königreichs Sachsen, des Rheinlands und Westfalens auf. Dadırch wird die alte Erfahrmng bestätigt, daß da, wo die Aerzteschaft auf Krankenkassenbevölkerung allein angewiesen ist, nur wenige Aerzte ihr Auskommen finden können.

Die Zahl der Aerztinnen nimmt in Deutschland beträchtlich zu, sie beträgt jetzt 195 ind war in den Jahren

$\begin{array}{rrrr}1908 & 55 & 1911 & 118 \\ 1909 & 69 & 1912 & 151 \\ 1910 & 102 & 1913 & 195\end{array}$

Sie suchen mit Vorliebe die Großstädte anf; nach dem Reichsmedizinalkalender hielten sich 1913138 Aerztinnen $(=70,8 \%$ ) in ihnen auf. In den folgenden Großstädten war ihre Zahl

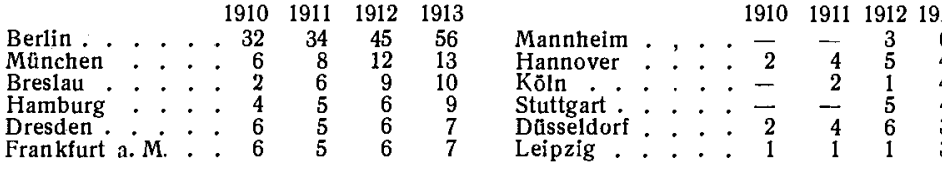

Von den Aerztimnen des Jahres 1913 kamen auf das östliche Preußen (mit Berlin) 79, auf das westliche 43, auf das ubrige Norddeutschland 14 auf Sachsen 14, auf Bayern 20, auf Wïrttemberg 8, auf Baden 14, anf Hessen 2 Aerztinnen, auf Elsaß-Lothringen 1. In Oesterreich waren Ende 1912 unter 13599 Aerzten 125 solche weiblichen Geschlechts (gegen 100 im Vorjahre). Die Zahl der weiblichen Medizinstudierenden nimmt in Deutschland von Jahr zu Jahr zu; ihre Zahl (mit Einrechnıng der Ausländerinnen) war

$$
\begin{array}{ccc} 
& \text { im Sommerhalbjahr } & \text { im Winterhalbjahr } \\
1911 & 510 & 566 \\
1912 & 622 & 683 \\
1913 & 773 & -
\end{array}
$$

Besondere Beachtung verdient die Entwicklung des Spe zialistentums. Dieses blïht hauptsächlich in den Großstädten, doch fehlt es anch in den Mittelstädten nirgends an Spezialärzten. Eine große Zahl von Aerzten, die für sich ein Spezialfach in Anspruch nehmen, übt allerdings nebenbei allgemeine Praxis aus, wenn auch mehr und mehr die Anschauung durchdringt, daß nur die Aerzte, die anf letztere verzichten, ein Anrecht auf den Namen Spezialarzt hahen. Bei der folgenden Auszählung der Spezialfächer sind die selteneren Kombinationen nicht aufgenommen worden, um das Verzeichnis nicht zu sehr zu verlängern, so z. B. Magenleiden und innere Krankheiten, Kinderkrankheiten und Gynäkologie, Hautkrankheiten 1md Gynäkologie etc.; diese Kombinationen, die nie in größerer Anzahl vorkommen, sind der Spezialität zugeschrieben worden, die als die wichtigere erschien. Die stehen.

1) Im Artikel für 1912 blieb infolge eines Schreibfehlers 14981
Aerzte für Lungenleiden sind denen für innere Krankheiten zugezählt. Mit den Lungenleiden befassen sich auch manche Kehlkopfspezialisten, sie sind diesen zugerechnet. In der folgenden Tabelle findet sich die Besetzung der verschiedenen Spezialfächer in den Großstädten:

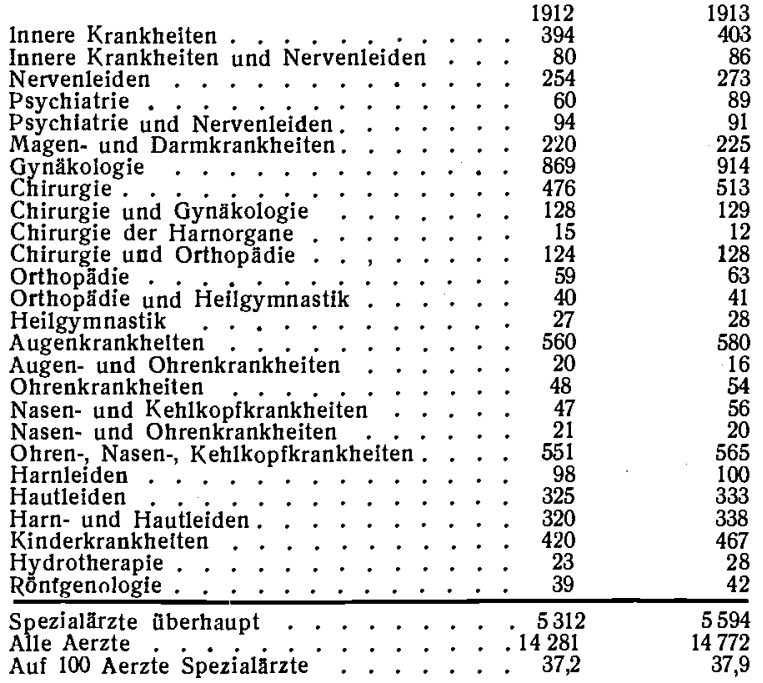

Unter den Spezialärzten stehen die Gynäkologen der Zahl nach weitaus an erster Stelle. Vereinigt man die Chirurgen mit den Aerzten, die Chirurgie in Verbindıng mit Gynäkologie und Orthopädie ausüben, so kommen sie an Zahl gleich nach den Gynäkologen, dann folgen die Augenärzte, die Ohren-, Nasen- und Kehlkopfärzte, endlich die Spezialärzte für Haut- und Harnleiden und die Kinderärzte. Eine genane Reihenfolge läßt sich nicht aufstellen, da sehr viele Spezialärzte mehrere Spezialfächer zugleich betreiben. Von Röntgenologen sind nur die anfgeführt, bei denen kein anderes Spezialfach angegeben ist.

In den Großstädten war 1912 und 1913 der Prozentsatz der Spezialärzte iuter der Gesamtzahl der Aerzte

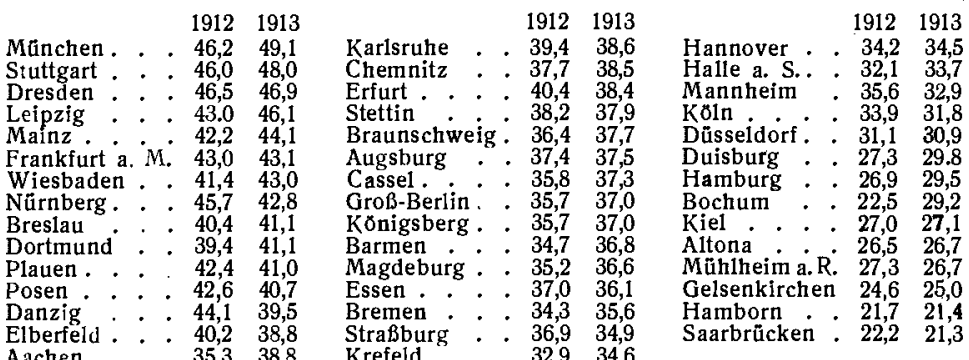

Man trifft hinsichtlich des Prozentsatzes der Spezialärzte große Verschiedenheiten, die von Gewohnheiten und dem Wohlstand der Bevölkerung, von der Größe des Hinterlandes, von den Gepflogenheiten der ärztlichen Vereinigungen und ähnlichen Dingen abhängen. In einer Anzahl von Großstädten hat der Prozentsatz der Spezialärzte erheblich zugenommen, so in München, Stıttgart, Mainz, Dortmınd, Groß-Berlin, Barmen, Dinisburg und Bochum.

Am 13. Mai 1913 waren es 30 Jahre, daß das erste Gesetz ïber die Krankenversicherung der Arbeiter vom Reichstag angenommen wurde, in welchem so wenig wie in den späteren Gesetzen die Stellung der Aerzte zu den Krankenkassen geregelt war. Die wirtschaftliche und kulturelle Entwicklung Deutschlands in diesen drei Jahrzehnten hat alle Erwartungen übertroffen; auf dem Gebiete der ärztlichen Wissenschaft, insbesondere auf dem der Hygiene, hat Deutschland, wie auf so manchen anderen, die Fiihrerschaft übernommen. Die Regierungen scheuen keine Geldopfer, die medizinischen Einrichtungen der Hochschnlen anf voller Höhe zu erhalten nnd dadurch den Studierenden Gelegenheit zı geben, die neuen und großen Fortschritte der ärztlichen Wissenschaft sich zu eigen zu machen. Zngleich wurden die Anforderungen bei den Prüfungen beträchtlich erhöht, Hygiene und Psychiatrie wurden in diese anfgenommen, und $1 \mathrm{~m}$ den angehenden Arzt vor der Erteilung der Approbation noch mehr an das Krankenbett zi bringen, wurde das Praktikantenjahr eingeführt. Allbekannt ist, wie eifrig die Studierenden das, was ihnen von den Hochschulen geboten wird, ausnutzen, nnd daß gar viele nach dem Praktikantenjahr durch Uebernahme von Assistentenstellen ihre weitere Ausbildung zu heben suchen. Man wird nirgends auf Widerspruch stoßen, wenn man sagt, daß der deutsche Aerztestand nach seinem Kömen und Wissen auf hoher Stufe steht.

Da erhebt sich von selbst die Frage: Entspricht dieser gründlichen, langjährigen, für den Studierenden und für den Staat so teuren ärztlichen Ausbildung die Stellung, die der Arzt heute im öffentlichen Leben einnimmt? Diese Frage muß entschieden verneint werden, jedenfalls für den größten Teil derjenigen Aerzte, die auf Kassenpraxis angewiesen sind. Und deren sind sehr viele. 
Man hat dies der großen Zunahme der Zahl der Aerzte zugeschrieben. Tatsächlich sind $\mathrm{ja}$ in den Städten sehr viele Aerzte zusammengedrängt, worauf in diesen Berichten oft hingewiesen wurde. Auf dem Lande ist allerdings da und dort über Mangel an Aerzten geklagt worden; aber diese Klagen sind nur zum kleinsten Teil berechtigt. Da, wo auf dém Lande die Industrie vorherrscht, ist die Arbeiterbevölkerung häufig auf die festangestellten Kassenärzte angewiesen; einem Nichtkassenarzt ist es ganz unmöglich, sich daneben eine Praxis zu schaffen. So sehen wir in der Rheinprovinz, in Westfalen, in Schlesien, in Sachsen, dah nicht so selten große Ortschaften von 10000-20000 Seelen auf einen einzigen Arzt angewiesen sind. Wo es sich auf dem Lande um eine rein agrarische Bevölkerung handelt, findet der Arzt wegen der geringen Dichte der Bevölkerung und bei der Neigung der Bauernschaft, den Arzt nur im äußersten Notfall zu Rate zu ziehen, sehr oft kein genügendes Auskommen.

Es wäre falsch, die unbefriedigende Stellung der Aerzte allein deren großer Zahl zuzuschreiben. Vor allem sind es nicht eigentlich die wirtschaftlichen Sorgen, die die Aerzte in den Kampf getrieben haben, es ist die beschämende Abhängigkeit des Aerztestandes von den Kassenvorständen, die wir überall sehen, wo das System der fixierten Kassenarztstellen herrscht. Diese Abhängigkeit muß durchbrochen werden. Es wäre niemals gelungen, wegen wirtschaftlicher Sorgen die Aerzteschaft zu dieser Einigkeit zusammenzuschmieden, die alle, wenn auch manche nur mit Schrecken, bewundern. Die Ideale, welche die Aerzte von der Hochschule mitbringen und die sie ihr ganzes Leben begleiten, ja begleiten müssen, wenn sie eine ersprießliche ärztliche Tätigkeit ausüben sollen, diese Ideale sind es, die der Aerztestand gefährdet sieht und die zu erhalten er alles daransetzen muß.

Mit Stolz werden die großen Erfolge der sozialen Gesetzgebung, insbesondere die der vorbeugenden Heilbehandlung, von den Regierungen immer wieder hervorgehoben. Mit vollem Recht. Nur selten wird aber derjenige genannt, der diese Erfolge in erster Linie ermöglicht hat, der Aerztestand. Man nehme die Erfolge der Lungenheilstätten. Wie fast endloses Belehren, Ermahnen, Zureden gehörte in den ersten zehn Jahren dazu, die Kranken zu einer Heilstättenkur zu bewegen, und war das gelungen, so waren die Eltern, die Gattin, die Anverwandten zu beruhigen! Auch vor Einführung der Familienbehandlung hatte der Arzt, der jetzt viel häufiger, als dies früher der Fall war, in die Arbeiterfamilie kam, oft Gelegenheit, die Mütter über Säuglingsernährung, über Verhalten bei ansteckenden Krankheiten der Kinder, über allerlei Dinge der täglichen Hygiene zu belehren, er hat niemals gezögert, sein Wissen nitzuteilen, ohne auf nennenswerten Entgelt rechnen zu können. Ueberaus segensreich war diese Kleinarbeit des Arztes in den 30 Jahren des Bestehens der Krankenversicherung; freilich läßt sie sich nicht in Zahlen ausdrücken, und darum geht die große Menge achtlos daran vorüber. Aber die Regierungen hätten diese 30 jährige Arbeit des Arztes richtig einschätzen können. Leider haben dies die Organe der Reichsregierung und die der meisten Regierungen der Bundesstaaten nicht getan. So wurde den Aerzten ein Kampf aufgezwungen, der so leicht zu vermeiden gewesen wäre, wenn die Forderungen der Aerzte, die, ohne Vorurteil betrachtet, so überaus gering sind, durch die Regierungen Unterstïtzung gefunden hätten. 